\title{
Quantum dots-based tissue and in vivo imaging in breast cancer researches: current status and future perspectives
}

\author{
Lin-Wei Wang ${ }^{1} \cdot$ Chun-Wei Peng ${ }^{1} \cdot$ Chuang Chen ${ }^{2} \cdot{\text { Yan } \mathrm{Li}^{1}}^{1}$
}

Received: 25 January 2015/Accepted: 26 March 2015/Published online: 2 April 2015

(C) The Author(s) 2015. This article is published with open access at Springerlink.com

\begin{abstract}
As the most common malignant tumor for females, breast cancer (BC) is a highly heterogeneous disease regarding biological behaviors. Precisely targeted imaging on $\mathrm{BC}$ masses and biomarkers is critical to $\mathrm{BC}$ detection, treatment, monitoring, and prognostic evaluation. As an important imaging technique, quantum dots (QDs)-based imaging has emerged as a promising tool in $\mathrm{BC}$ researches owe to its outstanding optical properties. However, few reviews have been specifically devoted to discussing applications of QDs-based imaging in BC researches. This review summarized recent promising works in QDs-based tissue and in vivo imaging for BC studies. Physicochemical and optical properties of QDs and its potential applications were briefly described first. Then QDs-based imaging studies in BC were systematically reviewed, including tissue imaging for studying biomarkers interactions, and evaluating prognostic biomarkers, in vivo imaging for mapping axillary lymphatic system, showing BC xenograft tumor, and detecting $\mathrm{BC}$ metastases. At last, the future perspectives with special emphasis on the potential clinical applications have also been discussed. Potential applications of QDs-based imaging on clinical BC in the future are mainly focused on tissue study, especially in BC molecular pathology due to its optimal optical properties and quantitative information capabilities on multiple biomarkers.
\end{abstract}

Yan Li

liyansd2@163.com

1 Hubei Key Laboratory of Tumor Biological Behaviors and Hubei Cancer Clinical Study Center, Department of Oncology, Zhongnan Hospital of Wuhan University, No 169, Donghu Road, Wuchang District, Wuhan 430071, Hubei Province, China

2 Department of Breast and Thyroid Surgery, Renmin Hospital of Wuhan University, Wuhan, China
Keywords Breast cancer - Quantum dots · Imaging · Tissue $\cdot$ In vivo

$\begin{array}{ll}\text { Abbreviations } \\ \text { BC } & \text { Breast cancer } \\ \text { QDs } & \text { Quantum dots } \\ \text { MRI } & \text { Magnetic resonance imaging } \\ \text { NIR } & \text { Near-infrared } \\ \text { ALS } & \text { Axillary lymphatic system } \\ \text { HER2 } & \text { Human epidermal growth factor receptor-2 } \\ \text { ER } & \text { Estrogen receptor } \\ \text { PR } & \text { Progesterone receptor } \\ \text { ALN } & \text { Axillary lymph nodes } \\ \text { SLN } & \text { Sentinel lymph node } \\ \text { PEG } & \text { Polyethylene glycol }\end{array}$

\section{Introduction}

Breast cancer (BC) is the most common malignant tumor for females worldwide [1]. With the advancement in screening tools and comprehensive therapies for BC, considerable improvements have been achieved over the past few decades [1-3]. It has been widely accepted that $\mathrm{BC}$ is a highly heterogeneous disease, with different biological behaviors for the same stage of $\mathrm{BC}$ patients [4]. Therefore, personalized medicine determined by multiplex information on prognosis is the main direction for BC therapy in the future. For individualized cancer therapy, cancer imaging including macroscopic cancer imaging techniques (computed tomography, magnetic resonance imaging, MRI; positron emission tomography and ultrasonography) and microscopic cancer imaging techniques (hematoxylin- 
eosin, immunohistochemistry, and immunofluorescence) play critical roles in cancer detection, treatment, prognosis evaluation, and disease course monitoring.

Preventive and predictive oncology will be the direction of future clinical oncology, which is different from traditional therapeutic oncology $[5,6]$. The essence is to obtain adequate information on prognosis from tumor tissues, and to predict future biological behaviors of tumor. Eventually, specific personalized treatment strategies are formulated based on the integrated prognostic information. In this regard, traditional imaging techniques could not satisfy the need for acquiring more specific and unique information on $\mathrm{BC}$ biology. For example, it has been well recognized that tumor invasion and metastasis are closely associated with coevolution of cancer cells and tumor microenvironment, which involve multiple biomarkers [7, 8]. However, how to simultaneously obtain multi-dimensional information from both cancer cells and tumor microenvironment is a formidable challenge for traditional imaging techniques. Therefore, new imaging techniques to reveal multi-dimensional information clearly and precisely are urgently needed in cancer research.

Recently, nanotechnology has emerged as a promising tool in biomedicine research. An important branch of nanotechnology is optical-based nanoparticles imaging, such as quantum dots (QDs)-based imaging, which has showed promising potential applications in cancer research $[9,10]$. The majority of QDs are semiconductor nanocrystals with many better optical properties than traditional organic dyes, such as high fluorescence intensity, strong resistance to photobleaching and chemical degradation, size-tunable emission wavelength and, large twophoton cross section, simultaneous multiple fluorescence under a single excitation source [9, 11-13]. Because of these optical advantages, QDs-based imaging has been widely applied in cancer researches. This review summarizes the current status and future perspectives of QDsbased tissue and in vivo imaging in $\mathrm{BC}$ researches, with special emphasis on the potential clinical applications.

\section{Physicochemical and optical properties of QDs}

The majority of QDs are semiconductor nanocrystals with core size ranging from 2 to $10 \mathrm{~nm}$, composed of two types of atoms from the II-VI group elements of periodic table of chemical element $[11,12,14,15]$. When QDs are excited by an external high-energy light, the internal electron of QDs will transform from its ground state to a higher level. The high-level electron relaxes and returns to the ground state, a photon is emitted producing fluorescence $[11,12$, $16,17]$. The minimal energy required to excite an electron from its ground state to a higher level is called as band gap energy, which is dependent on the size of the complex, the larger size, the smaller the band gap. Therefore, QDs have an advantage of continuous and size-tunable emission wavelength ranging from whole visible light to mid-infrared light regions $(400-5000 \mathrm{~nm})[16,18,19]$. QDs also has narrow emission and wide excitation spectrum advantages, a property favoring multiplexed imaging under oneexcitation spectrum without mutual interference among targeted signals $[11,12]$. Because of the small size, the entire QDs particle could behave like a single molecule with the component atoms exciting and emitting light simultaneously to produce high signal intensity in the form of strong fluorescence [12, 16, 17, 20].

For tumor applications, QDs are encapsulated with diverse types of amphiphilic polymers to make it more biocompatible and water-soluble [16, 17]. Those amphiphilic polymers often carry chemically active groups, such as carboxyl, hydroxyl, and thiol groups, which can couple with modified functional agents, such as streptavidin, drug, small molecule, DNA, and antibody. The modified QDs could be used for tumor studies, such as biomarker detection [21, 22], tumor imaging [23, 24], tumor heterogeneity demonstration [25-27], cancer diagnosis [16], treatment [28], and monitoring [16, 29] (Fig. 1A).

\section{Applications of QDs-based imaging in BC}

One specific application of QDs is that it has a narrow emission spectrum but a size-dependent tunable emission wavelength ranging from visible to near-infrared (NIR) light, and this unique property makes QDs-based imaging widely applicable for tissue and in vivo BC studies. For tissue studies, QDs-based imaging has been used to study biomarkers interactions (Fig. 1B) and evaluate prognostic biomarkers (Fig. 1C). For in vivo studies, QDs-based imaging has been applied to map axillary lymphatic system (ALS) (Fig. 1D), show BC xenograft tumor (Fig. 1E), and detect $\mathrm{BC}$ metastases (Fig. 1F).

\section{QDs-based imaging to study biomarkers interactions}

It has been well recognized that malignant biological behaviors of cancer are due to the interactions of key molecules from both tumor cells and microenvironment [7, 8]. Therefore, it is important to develop a method to simultaneously demonstrate the interactions of different molecules, so as to decipher the tumor biological behaviors from a multivariate perspective instead of a univariate perspective. Currently, many traditional methods are available to obtain a single biomarker information at one time, such as immunohistochemistry, immunofluorescence, 


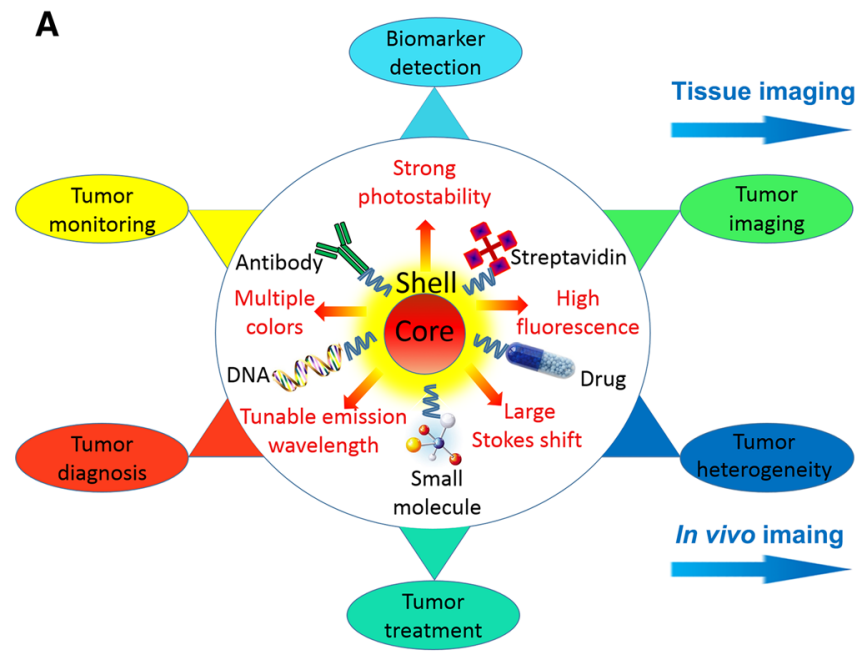

Fig. 1 Optical properties and potential applications of QDs in BC researches. Commonly used QDs are core-shell structure encapsulated with amphiphilic polymers carrying chemically active groups. Compared with traditional organic dyes, QDs show excellent optical properties (A). After being coupled with active molecules, QDs can

and Western blotting. These methods, however, share one common drawback, that is they cannot obtain in situ quantitative information with morphological features for multiple biomarkers.

One encouraging progress in this field is the development of QDs-based multiplexed imaging that shows enormous potentials for in situ multiplexed imaging to simultaneously reveal the interactions of different molecules $[9,30]$. The technical procedures of QDs-based immuno-conjugation plus biotin-streptavidin imaging on single biomarker and double biomarkers are illustrated in Fig. 2A1 and A2. In our study, QDs-based quantitative and in situ multiplexed imaging on human epidermal growth factor receptor-2 (HER2) and estrogen receptor (ER) of BC tissues was developed, which may help understand the evolutionary process of $\mathrm{BC}$ heterogeneity [30]. For example, it has been well known that HER2 level in BC is closely related to $\mathrm{BC}$ progression, but direct visual evidence from tissue level is lacking to support the conclusion. QDs-based multiplexed imaging on HER2 and IV collagen was applied in our study, and demonstrated the invasive behaviors of $\mathrm{BC}$ by progressive degradation and destruction IV collagen with the increase of HER2 expression level in $\mathrm{BC}$ tissue, especially at the invasion edge (Fig. 2B1-B4) [31]. Based on QDs imaging on IV collagen, patterns of tumor invasion were described as washing pattern, ameba-like pattern, polarity pattern, and linear pattern (Fig. 2C1-C4) [32]. These patterns clearly show interactions between cancer cells and microenvironment during different invasive periods. In addition, it has been

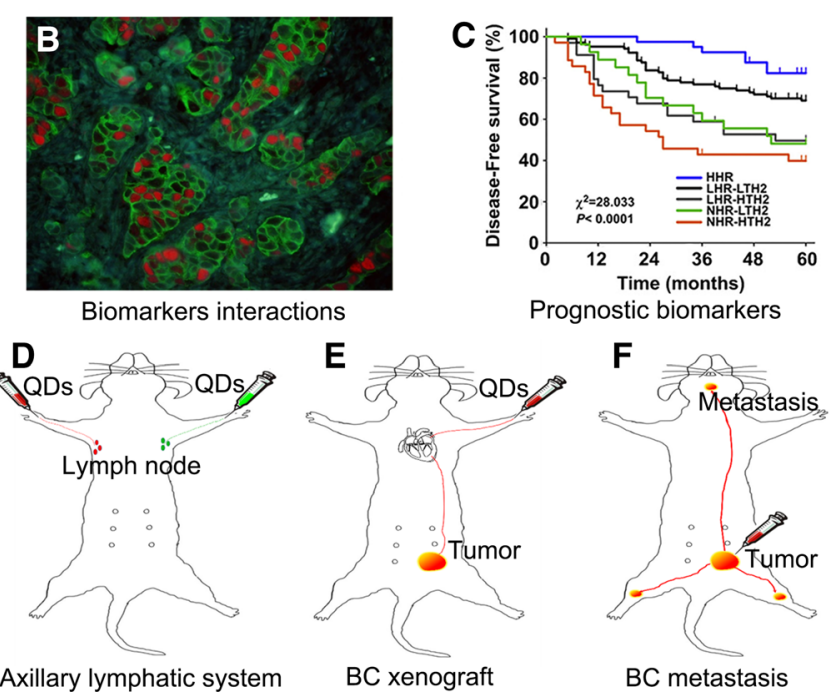

be adapted for tissues imaging, such as studying biomarker interactions (B) and evaluating prognostic biomarkers (C), and for in vivo imaging such as mapping ALS (D), showing BC xenograft (E) and detecting $\mathrm{BC}$ metastasis $(\mathbf{F})$ in $\mathrm{BC}$ researches

widely recognized that tumor microenvironment is a powerful stimulation factor for cancer invasion and metastasis. Interestingly, further study showed dynamic changes in IV collagen during cancer invasion, which implied the pulse-mode of cancer invasion and metastasis (Fig. 2D1, D2) [33]. Yet, few studies could directly observe the co-expression and dynamics of multiple biomarkers in tumor microenvironment. In addition, QDsbased multiplexed imaging has also been employed to simultaneously reveal the dynamic interactions between biomarkers in tumor microenvironment (IV collagen, tumor angiogenesis and infiltrative macrophages) and cancer cells (matrix metalloproteinase 9), by incorporating the temporal and spatial dimensions [34].

\section{QDs-based imaging to evaluate prognostic biomarkers}

Cancer outcome is the most important concern for oncologists and patients. TNM staging system is the universal language to determine the clinical stages of cancer, predict the prognosis, and guide the treatment options. For this staging system, $\mathrm{T}$ describes primary tumor size and whether it has invaded nearby tissue, $\mathrm{N}$ describes nearby/regional lymph nodes that are involved, and $\mathrm{M}$ describes distant metastasis [35]. Currently, lymph node-negative BC patients have been the majority of the new cases [36]. And, with the advancement of mass screening programs and improved detection facilities, the proportion of early $\mathrm{BC}$ has been on steady increase over the past two decades. For 

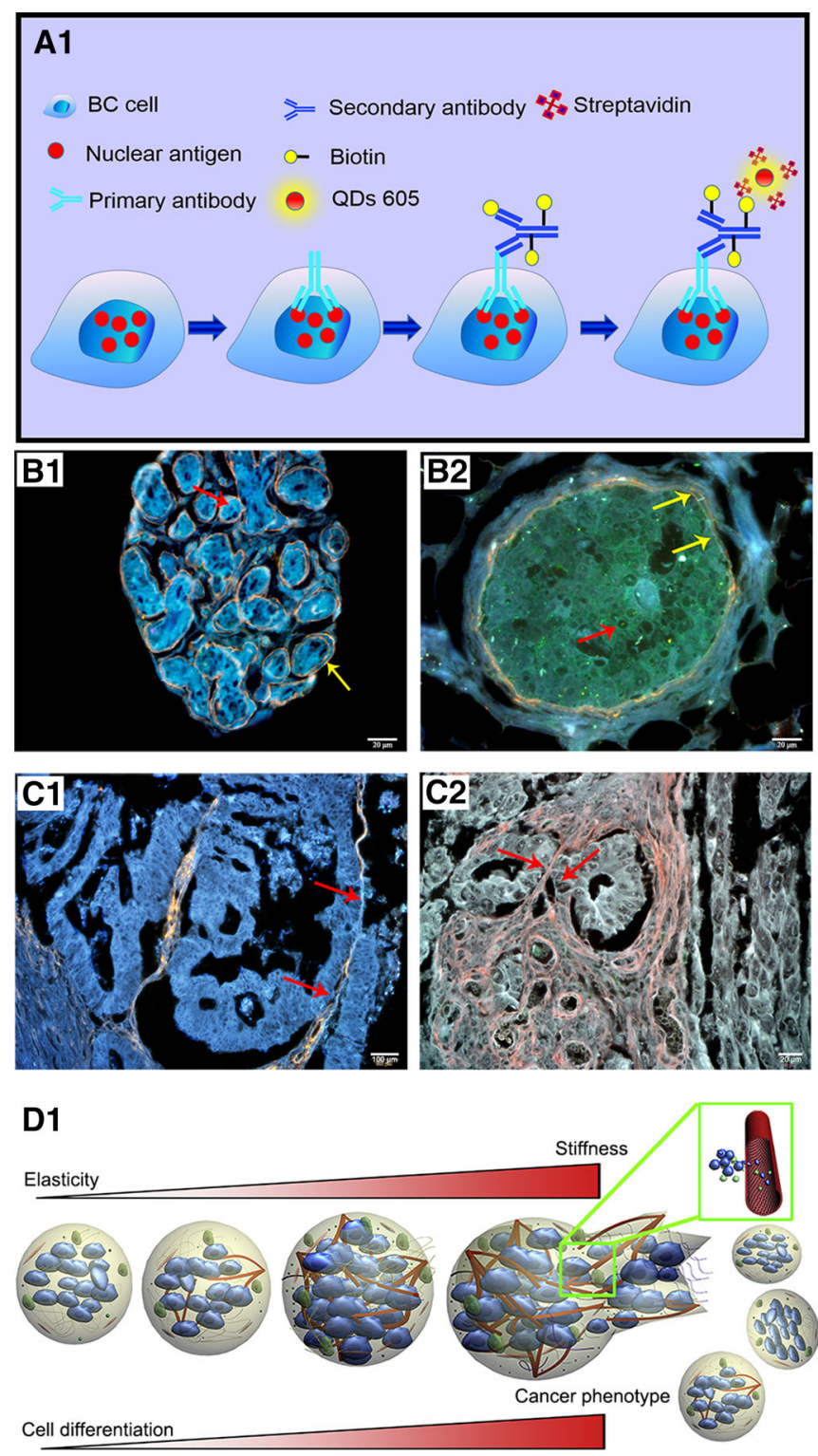

Fig. 2 QDs-based imaging for studying BC biomarker interactions. Schematic plots of QDs-based single (A1) and double biomarkers imaging (A2). Decrease of IV collagen (yellow arrows) with the increase of HER2 (red arrows): HER2 (0), intact IV collagen (B1); HER2 (+), IV collagen becomes unsmooth and thinner (B2); HER2 $(2+)$, IV collagen becomes degraded (B3); HER2 (3+), complete IV collagen degradation (B4). BC invasion patterns: Washing Pattern

those, early $\mathrm{BC}$ with both $\mathrm{N}$ and $\mathrm{M}$ information negative, TNM staging system is no longer efficient as an informative tool to predict prognosis and guide treatment option [5]. Therefore, much information closely related with $\mathrm{BC}$ prognosis should be obtained from the $\mathrm{BC}$ tumor mass itself $[5,36]$.

With the development of molecular biology, many prognostic biomarkers hidden in BC mass have been discovered. Specific labeling and accurate quantification of
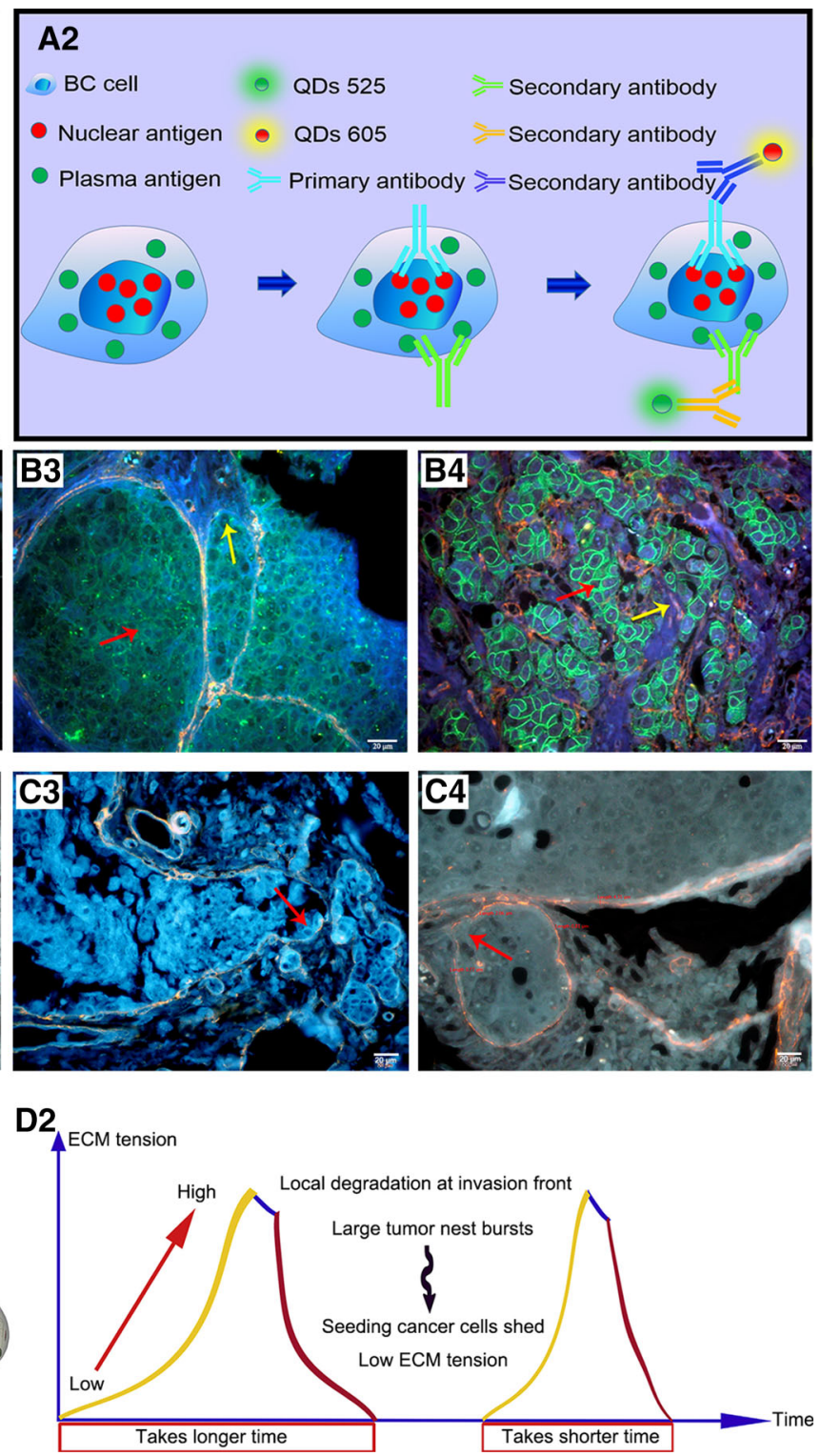

(C1), Ameba-like Pattern (C2), Polarity Pattern (C3), and Linear Pattern (C4). Dynamic changes of IV collagen implied the pulsemode of cancer invasion and metastasis: expansive growth of tumor nests burst to form disseminations (D1), the process from growth to burst is more quickly than the last (D2). ECM: extracellular matrix. Reproduced with permission from Ref [31-33]

those prognostic biomarkers are the key procedure to evaluating $\mathrm{BC}$ prognosis. In our previous studies, QDsbased imaging and quantitative spectral analysis on biomarkers of $\mathrm{BC}$ were developed and showed good correlation and consistency with conventional IHC, with better image quality and sensitivity $[25,37,38]$. By this method, a new indicator, total HER2 load was obtained integrating information on BC tumor size and QDs-based HER2 quantification, which could better reveal $\mathrm{BC}$ heterogeneity 
and new subtypes than current methods [26]. Furthermore, a new molecular classification system, 5 molecular subtypes based on separate spectral quantification of HER2, $\mathrm{ER}$, and progesterone receptor (PR) was established (Fig. 3) [27]. Significantly, this classification system by simple QDs-based imaging was as informative as multigenes analysis with lower cost. Consequently, this method could have more potentials in clinical applications than multi-gene assays, especially in developing countries where multi-genes analysis is expensive for patients [27].
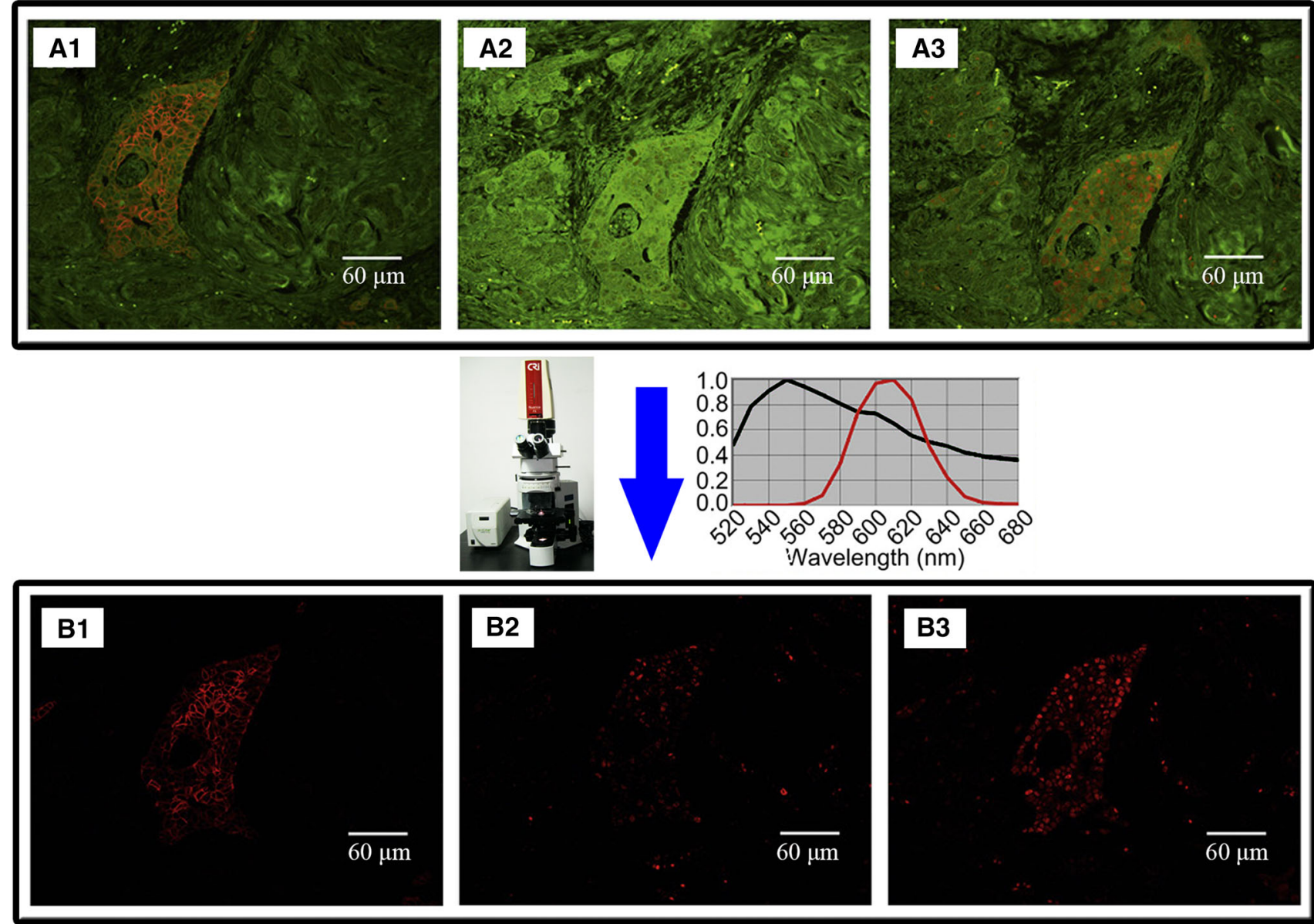

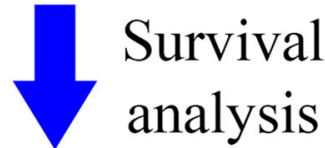
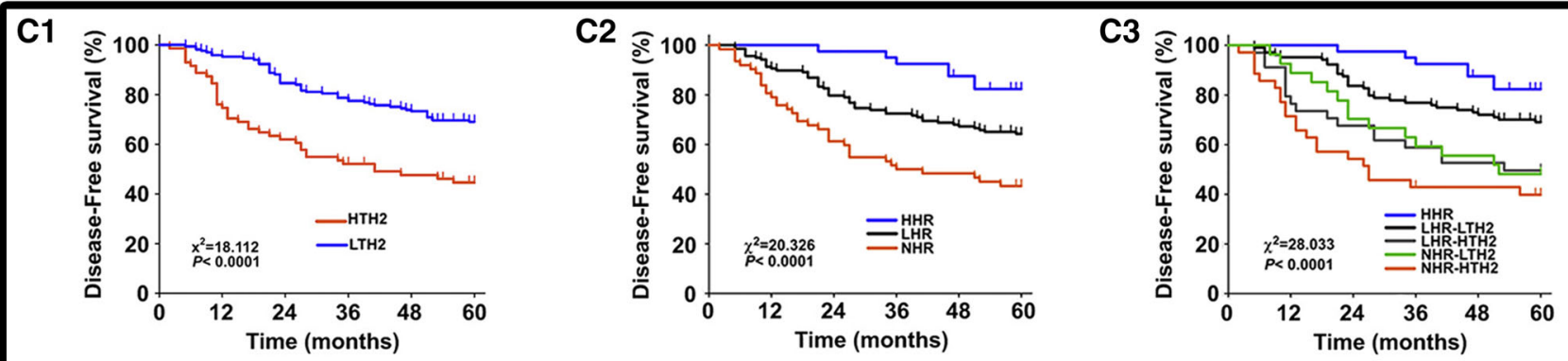

Fig. 3 QDs-based imaging for evaluating $\mathrm{BC}$ prognostic biomarkers. QDs-based imaging on HER2 (A1), ER (A2), and PR (A3) was acquired, unmixed, and quantified by CRi Nuance software. Unmixed images of HER2 (B1), ER (B2), and PR (B3). Survival analysis on HER2 load (C1), hormone receptors (C2), and 5 molecular subtypes
(C3). HTH2: high total HER2 load; LTH2: low total HER2 load; HHR: high hormone receptors; LHR: low hormone receptors; NHR: negative hormone receptor. Reproduced with permission from Ref $[26,27]$ 
These studies demonstrated that QDs-based multiplexed imaging may be a promising strategy for quantitative and more accurate diagnostic pathology.

\section{QDs-based imaging to map ALS}

ALS is the most important lymphatic drainage system and main lymphatic metastasis routes of BC. Axillary lymph nodes (ALN) dissection is performed to eliminate potential lymphatic metastasis and to evaluate prognosis of $\mathrm{BC}$. Presently, lymph node-negative $\mathrm{BC}$ has been on the steady increase worldwide, and minimal invasive surgery has been advocated widely in clinical practices [36]. Under these circumstances, ALN dissection is not a preferred operation because it is not necessary in most BC patients and its complications (postoperative upper limb edema and other dysfunctions) is common in BC patients. Instead, increasing attention has been focused on sentinel lymph node (SLN) which is the first and inevitable site when lymph node metastasis occurs in BC [39]. Detection of SLN is enough to evaluate ALN status, as against systematic clearance. Therefore, preoperative and intraoperative detection and imaging of ALS is the key procedure in predicting lymph metastasis, designing surgical plan, and evaluating prognosis.

Optical imaging is an important method to detect and image lymph system. Compared with visible light, NIR fluorescence has advantages in imaging lymph system due to its high tissue penetration through the skin and subcutaneous tissues. NIR QDs with an emission wavelength ranging from 700 to $2500 \mathrm{~nm}$ are suitable to map lymphatic system because tissue penetration depth of NIR QDs $>5 \mathrm{~mm}$, which can penetrate the skin and subcutaneous tissues to realize lymph imaging in large animal (such as pig). Simultaneously, QDs has a larger two-photon cross section than conventional fluorescent dyes which can avoid the interference of tissue autofluorescence and can help achieve deep tissue imaging [13, 20]. Using such NIR QDs, Kim et al. [24] first achieved mapping axillary SLN of mouse and femoral SLN of pig. Many similar studies attempted to map lymphatic system have been conducted thereafter (Table 1).

A potential advantage of QDs-based imaging is that it can simultaneously assess and distinguish lymphatic drainages from multiple separate drainage areas, which is difficult for traditional lymphangiography techniques, such as X-ray, MRI, or radionuclide [47-49]. Hama et al. [47] adopted two NIR QDs with different wavelengths to simultaneously map two lymphatic flow pathways from the breast and the upper extremity. This method could visualize lymphatic drainage territories of the two different areas as well as lymphatic vessel in real time. Subsequently, as many as 5 separate drainage areas of lymph nodes were successfully imaged by 5 kinds of QDs with different emission spectra in mice models $[43,46]$. In a study by Kobayashi et al. [49], QDs-labeled melanoma cells and optically labeled dendrimers were used to simultaneously visualize migration of melanoma cells and the lymphatics in vivo. This method can provide a noninvasive in vivo imaging to demonstrate the relationship between cell migration and lymphatic drainage patterns. Additionally, a strong fluorescence signal in SLN was detected within several minutes after QDs injection and maintained for over $24 \mathrm{~h}$ [44]. Durable and strong SLN fluorescence makes it easier for detection and subsequent managements (i.e., biopsy and dissection) of SLN. Although there have been concerns that in vivo application of QDs may cause nonspecific targeting to other tissues or organs because the reticuloendothelial system could take QDs [50], nonspecific targeting is not found in QDs-based ALS imaging of BC [44].

\section{QDs-based imaging to show BC xenografts}

The optical advantages of QDs have also been applied to target and image tumor xenografts (Table 2). In vivo prostate cancer targeting and imaging by QDs was reported by Gao et al. as early as 2004 [23]. We have also described targeting and imaging of QDs coupled with alpha-fetoprotein monoclonal antibody in human hepatocellular carcinoma xenografts of nude mouse as well as its lung metastasis [51]. Similarly, QDs also could be conjugated with biomarker of $\mathrm{BC}$ to develop an ideal probe for in vivo targeting and imaging in BC. A study of Rizvi et al. [52] demonstrated the successful applications of NIR QDsconjugated HER2 in fixed and live HER2-overexpressed BC cells. In animal models, QDs-conjugated anti-HER2 antibody was applied to image HER2-overexpressed BC xenografts and observed the delivery process of QDs from blood circulation to cancer cell perinuclear region [53]. Animal model studies also found that the fluorescence of QDs directly conjugated with anti-HER2 antibody was stronger than that of traditional polyethylene glycol (PEG)coated QDs in visualizing HER2-positive BC xenograft [54, 55]. In addition, complex of QDs and trastuzumab can achieve high-resolution 3-dimensional target imaging in BC-bearing mice [56]. Besides target imaging, the complex containing QDs and trastuzumab may also have therapeutic effect on BC. Targeted removal of tumor can help the precise excision of tumor with minimal damage on normal tissues. By the guidance of NIR QDs imaging on xenografted tumor, targeted removal of subcutaneous tumor was successfully performed [57]. Moreover, with the development of fluorescence-mediated tomography, the in vivo fluorescence imaging depth could reach as deep as $10 \mathrm{~cm}[58,59]$, which may be useful to guide intraoperative surgery for deep BC mass in the near future. 
Table 1 QDs-based imaging on ALS

\begin{tabular}{|c|c|c|}
\hline Researches & Study models & Major findings \\
\hline Helle et al. [40] & Mouse & $\begin{array}{l}\text { Visualization of SLN on right axilla was achieved both in healthy and BC-bearing mice with indium-based } \\
\text { NIR QDs, a toxicity-reduced tracer }\end{array}$ \\
\hline Pons et al. [41] & Mouse & $\begin{array}{l}\text { Cadmium-free QDs with reduced toxicity were synthesized to visualize right axillary and lateral thoracic } \\
\text { lymph nodes of mouse }\end{array}$ \\
\hline Pic et al. [42] & Mouse & $\begin{array}{l}\text { Comparison between fluorescence imaging and mass spectroscopy for detection of QDs-based imaging of } \\
\text { axillary and lateral thoracic SLN was performed, confirming QDs imaging could be a better strategy }\end{array}$ \\
\hline $\begin{array}{l}\text { Kosaka et al. } \\
\text { [43] }\end{array}$ & Mouse & $\begin{array}{l}\text { Five QDs with different emission spectra were injected at five different sites of mice to simultaneously show } \\
\text { multi-color lymphatic imaging of neck lymph nodes, thoracic lymph nodes, and ALN }\end{array}$ \\
\hline Robe et al. [44] & Nude mouse & ALN of nude mouse were identified by QDs and biological distribution of QDs in ALN was analyzed \\
\hline $\begin{array}{l}\text { Ballou et al. } \\
\text { [45] }\end{array}$ & Nude mouse & $\begin{array}{l}\text { Rapid migration of two PEG-coated NIR QDs from the tumor to surrounding lymph nodes (inguinal, axillary, } \\
\text { lumbar, and renal lymph nodes) was visualized in nude mice }\end{array}$ \\
\hline $\begin{array}{l}\text { Kobayashi et al. } \\
\text { [46] }\end{array}$ & Mouse & $\begin{array}{l}\text { Simultaneous imaging on deep cervical nodes, superficial cervical nodes, thoracic duct, lateral thoracic } \\
\text { nodes, and axillary nodes was achieved by } 5 \text { kinds of QDs with different emission spectra }\end{array}$ \\
\hline Hama et al. [47] & Mouse & $\begin{array}{l}\text { Simultaneously mapping two lymphatic flows from the breast and the upper extremity with two QDs under } \\
\text { an exciting light }\end{array}$ \\
\hline Kim et al. [24] & $\begin{array}{l}\text { Nude mouse } \\
\quad \& \text { pig }\end{array}$ & Imaging on femoral SLN of pigs and axillary SLN of mouse was achieved for the first time by NIR QDs \\
\hline
\end{tabular}

Table 2 QDs-based imaging on BC xenografts

\begin{tabular}{|c|c|c|}
\hline Researches & Study model & Major findings \\
\hline Ma et al. [61] & $\begin{array}{l}\text { Nude mice } \\
\text { mouse }\end{array}$ & $\begin{array}{l}\text { Anti-HER2 antibody was conjugated to the multilayered, core/shell nanoprobes containing magnetic ferric } \\
\text { oxide particles, visible-fluorescent QDs, and NIR QDs to simultaneously conduct MRI and fluorescence } \\
\text { imaging of BC-bearing nude mice }\end{array}$ \\
\hline $\begin{array}{l}\text { Balalaeva et al. } \\
\text { [54] }\end{array}$ & Nude mouse & $\begin{array}{l}\text { Fluorescent signal of QDs bound with anti-HER2 antibody was stronger than that of PEG-coated QDs in } \\
\text { visualizing HER2-positive BC xenograft }\end{array}$ \\
\hline Tan et al. [60] & Mouse & $\begin{array}{l}\text { A complex containing magnetic iron oxides and QDs was developed to perform multimodal image of MRI } \\
\text { and QDs in BC-bearing mice }\end{array}$ \\
\hline $\begin{array}{l}\text { Papagiannaros } \\
\text { et al. [65] }\end{array}$ & Mouse & $\begin{array}{l}\text { Anti-nucleosome antibody } 2 \mathrm{C} 5 \text { was coupled to NIR QDs containing polymeric micelles to enhance signal } \\
\text { intensity of BC tumor and melanoma lung pseudometastasis in mice }\end{array}$ \\
\hline $\begin{array}{l}\text { Papagiannaros } \\
\text { et al. [55] }\end{array}$ & Mouse & $\begin{array}{l}\text { PEG-phospholipid micelle-encapsulated QDs developed in this study showed more rapid speed and higher } \\
\text { fluorescence in imaging and quantifying BC tumor of mice than PEG QDs }\end{array}$ \\
\hline Park et al. [64] & Nude mouse & $\begin{array}{l}\text { A dual-modality complex contained magnetic iron oxide nanoparticles, QDs, and anti-cancer drug was } \\
\text { synthesized to simultaneously perform NIR imaging, MRI, and therapy on BC tumor }\end{array}$ \\
\hline Takeda et al. [56] & Mouse & $\begin{array}{l}\text { Complex of QDs and trastuzumab was injected to BC-bearing mice to perform in vivo target image by a } \\
\text { high-resolution } 3 \mathrm{D} \text { microscopic system }\end{array}$ \\
\hline Tada et al. [53] & Mouse & $\begin{array}{l}\text { QDs-conjugated with anti-HER2 antibody was injected to mice-bearing HER2-overexpressed BC to } \\
\text { analyze its delivery process from blood circulation to cancer cell perinuclear region }\end{array}$ \\
\hline
\end{tabular}

As an optimal probe, NIR QDs also can be linked with other imaging materials to develop a multimodality probe, which has multifunctional imaging capabilities in different imaging conditions. A multimodality probe containing magnetic iron oxides and QDs was developed by Tan et al. to perform dual-modal image of MRI and QDs in BCbearing mice [60]. Subsequently, a multilayered, core/shell nanoprobe containing ferric oxide (core), visible-fluorescent QDs (inner shell), and NIR QDs (outer shell) was fabricated by Ma et al. [61] to conduct in vivo multimodality imaging. With mean size of $150 \mathrm{~nm}$, competent magnetic property, and dual fluorescence at 600 and $750 \mathrm{~nm}$, this novel multilayered probe has been successfully conjugated with anti-HER2 antibody to achieve both targeted MRI and NIR imaging of BC tumor in nude mice. A dual-modality probe including QDs and magnetic Mn was designed by Ding et al. [62] to achieve fluorescence imaging and MRI of subcutaneous and intraperitoneal 
tumors in nude mouse, respectively. This probe opens a door for multimodality imaging and overcomes the shortcoming of QDs imaging in deep tissues. The heat produced by QDs when excited by laser irradiation can be used for highly sensitive imaging and selected destruction of deep tumor. QDs embedded in quantum well was applied by SalmanOgli et al. [63] to enhance sensitivity of thermal detection on small tumor based on the difference of temperature between tumor and normal tissue by computational model-based difference methods. Furthermore, a complex containing QDs, iron oxide, and doxorubicin was synthesized by Park et al. to perform QDs-based imaging, MRI, and therapy on BC-bearing nude mice [64].

\section{QDs-based imaging to detect $\mathrm{BC}$ metastasis}

Targeted imaging and early detection of metastasis, the major cause for cancer mortality, could help initiate effective therapy to improve patients' prognosis. Current imaging techniques are difficult to achieve early detection as we required, because those imaging techniques can detect a tumor only when the tumor cells grow up to change the structure of normal tissue. But QDs-based imaging could help achieve earlier detection by imaging on tumor cells, even single tumor cell in vivo [29]. In a study by Gupta et al. [66], BC cells were labeled with QDs to develop $\mathrm{BC}$ brain metastasis model of nude mice, which was used to demonstrate anti-metastatic effects of phenethyl isothiocyanate. By QDs-based imaging, this study demonstrated that phenethyl isothiocyanate not only suppress growth of brain metastasis, but also eradicate small metastasizes in other sides.

Micrometastasis is an early metastasis which takes place long before the development of obvious metastasis, and such micrometastasis within the diameter of 0.2 to $2.0 \mathrm{~mm}$ [35] is now considered as a powerful prognostic factor for BC [67]. As a result, rapid, sensitive, and accurate detection of micrometastasis holds important clinical significance in cancer therapy. At present, however, the unsatisfactory fact is that the conventional techniques, such as intraoperative frozen section examinations, often fail to reveal such micrometastasis because of low resolution. To tackle this problem, multi-color fluorescence imaging could provide promising solution, but low photostability and single-plexed imaging of traditional organic dyes limit the application of these fluorescent agents in clinical setting. In contrast, QDs could be developed to distinguish rare target cells from non-target tissues due to their high photostability and strong fluorescence intensity.

Another advantage of QDs-based imaging for micrometastasis of $\mathrm{BC}$ is that it can rapidly distinguish small metastasis from complex non-tumor tissues because of its strong fluorescence and target imaging [29, 68].
Comparison between QDs-based double-staining and rapid HE staining demonstrated that false-negative rate of QDsbased double-staining was much lower than HE staining in intraoperative diagnosis of $\mathrm{BC}$ lymph nodes micrometastasis and isolated tumor cells [68]. In terms of monitoring cancer cells metastasis process, QDs were also used to realtime monitoring the detail metastasis process of $\mathrm{BC}$ cells in nude mouse, which could observe the four initial steps of cancer metastasis: cancer cells far from blood vessels in tumor, near the vessel, in the bloodstream, and adherent to the inner vascular surface in the normal tissues near tumor [29]. Furthermore, a multimodality complex composed with iron oxide and QDs particle was used to label BC cells and track these cells in lymphatic system of mice with MRI and optical imaging system [69]. Importantly, single cancer cell within lymph nodes can also be clearly observed in real time.

\section{Limitations and future perspectives}

QDs have demonstrated enormous potentials in BC researches. However, some serious limitations including inherent toxicity, poor biocompatibility, and lack of multiplexed imaging and analytical systems should be systematically improved to further promote their use in cancer studies. Of these limitations, toxicity and biocompatibility are the main concerns, especially for in vivo study. Most of the currently used QDs contain heavy metal elements such as $\mathrm{Cd}, \mathrm{Te}, \mathrm{As}, \mathrm{Pb}$, and $\mathrm{Hg}[9,19,20]$, posing potential adverse effects on living systems. Although some studies revealed no toxic effect on culture cells and experimental animals [41, 51, 52], the long-term effect on living systems is unknown. A study by Chan et al. [70] indicated that the heavy metal core QDs could induce early-stage mouse blastocyst death both in vitro and in vivo. Furthermore, QDs can directly penetrate placental barrier to harm fetal development or even destroy fetuses, and the penetration behavior could not be eliminated by coating with PEG or $\mathrm{SiO}_{2}$ [71]. As an external material, QDs always accumulate into reticuloendothelial system (e.g., liver, spleen, and kidney) whose clearance rate from the body is very low $[51,71]$. Therefore, those QDs containing heavy metals should not be considered for in vivo application in clinical BC. Fortunately, some low-toxic or even non-toxic QDs, such as C-QDs, graphene QDs, silicon QDs, and silver QDs have been developed recently, which may avoid the heavy metals and have superior optical properties than traditional semiconductor heavy metalcontained QDs [10, 72, 73].

In the future, QDs will be used in $\mathrm{BC}$ researches more frequently and widely because of its excellent optical properties. With the advancement of fluorescence-mediated 
tomography, tumor located in deep tissue $(>10 \mathrm{~cm})$ will be revealed more clearly $[58,59]$, which may fully satisfy the need for imaging in BC. In terms of tissue study, QDsbased imaging had showed its optimal applicability in imaging acquisition and quantification of single and multiple biomarkers. So QDs-based imaging on tissues could be the main direction for clinical applications. By QDsbased imaging, co-expression, and coevolution of multiple biomarkers in cancer cells and microenvironment can be well studied. Simultaneously, prognostic values of those biomarkers could also be more accurately evaluated, which will effectively push forward the progress of molecular pathology and promote the transition to preventive and predictive oncology.

\section{Conclusions}

As an optimal imaging technique, QDs-based imaging has opened up a new field in $\mathrm{BC}$ research. Potential applications of QDs-based imaging on $\mathrm{BC}$ in the future are mainly focused on tissue study, especially in BC molecular pathology due to its optimal optical properties and quantified information abilities on multiple biomarkers.

Acknowledgments This work is supported by Project of the National Natural Science Foundation of China (81230031/H18, 81201196/H18, 81401515/H1819) and Fundamental Research Fund for Central Universities (303274028).

Conflict of interest The authors declared no conflict of interest.

Open Access This article is distributed under the terms of the Creative Commons Attribution-NonCommercial 4.0 International License (http://creativecommons.org/licenses/by-nc/4.0/), which permits any noncommercial use, distribution, and reproduction in any medium, provided you give appropriate credit to the original author(s) and the source, provide a link to the Creative Commons license, and indicate if changes were made.

\section{References}

1. Jemal A, Bray F, Center MM et al (2011) Global cancer statistics. CA Cancer J Clin 61:69-90

2. DeSantis C, Ma J, Bryan L et al (2014) Breast cancer statistics, 2013. CA Cancer J Clin 64:52-62

3. Siegel R, DeSantis C, Virgo K et al (2012) Cancer treatment and survivorship statistics, 2012. CA Cancer J Clin 62:220-241

4. Shackleton M, Quintana E, Fearon ER et al (2009) Heterogeneity in cancer: cancer stem cells versus clonal evolution. Cell $138: 822-829$

5. Wang LW, Qu AP, Yuan JP et al (2013) Computer-based image studies on tumor nests mathematical features of breast cancer and their clinical prognostic value. PLoS ONE 8:e82314

6. Hood L, Heath JR, Phelps ME et al (2004) Systems biology and new technologies enable predictive and preventative medicine. Science 306:640-643
7. Friedl P, Alexander S (2011) Cancer invasion and the microenvironment: plasticity and reciprocity. Cell 147:992-1009

8. Mao Y, Keller ET, Garfield DH et al (2013) Stromal cells in tumor microenvironment and breast cancer. Cancer Metastasis Rev 32:303-315

9. Chen C, Peng J, Sun SR et al (2012) Tapping the potential of quantum dots for personalized oncology: current status and future perspectives. Nanomedicine (Lond) 7:411-428

10. Cuenca AG, Jiang H, Hochwald SN et al (2006) Emerging implications of nanotechnology on cancer diagnostics and therapeutics. Cancer 107:459-466

11. Bruchez M Jr, Moronne M, Gin P et al (1998) Semiconductor nanocrystals as fluorescent biological labels. Science 281:2013-2016

12. Chan WC, Nie S (1998) Quantum dot bioconjugates for ultrasensitive nonisotopic detection. Science 281:2016-2018

13. Larson DR, Zipfel WR, Williams RM et al (2003) Water-soluble quantum dots for multiphoton fluorescence imaging in vivo. Science 300:1434-1436

14. Zrazhevskiy P, Sena M, Gao X (2010) Designing multifunctional quantum dots for bioimaging, detection, and drug delivery. Chem Soc Rev 39:4326-4354

15. Zrazhevskiy P, Gao X (2009) Multifunctional quantum dots for personalized medicine. Nano Today 4:414-428

16. Kairdolf BA, Smith AM, Stokes TH et al (2013) Semiconductor quantum dots for bioimaging and biodiagnostic applications. Annu Rev Anal Chem 6:143-162

17. Alam F, Yadav N (2013) Potential applications of quantum dots in mapping sentinel lymph node and detection of micrometastases in breast carcinoma. J Breast Cancer 16:1-11

18. Licha K, Olbrich C (2005) Optical imaging in drug discovery and diagnostic applications. Adv Drug Deliv Rev 57:1087-1108

19. Keuleyan S, Lhuillier E, Guyot-Sionnest P (2011) Synthesis of colloidal $\mathrm{HgTe}$ quantum dots for narrow mid-IR emission and detection. J Am Chem Soc 133:16422-16424

20. Smith AM, Duan H, Mohs AM et al (2008) Bioconjugated quantum dots for in vivo molecular and cellular imaging. Adv Drug Deliv Rev 60:1226-1240

21. Wu X, Liu H, Liu J et al (2003) Immunofluorescent labeling of cancer marker Her2 and other cellular targets with semiconductor quantum dots. Nat Biotechnol 21:41-46

22. Zhang H, Sachdev D, Wang C et al (2009) Detection and downregulation of type I IGF receptor expression by antibodyconjugated quantum dots in breast cancer cells. Breast Cancer Res Treat 114:277-285

23. Gao X, Cui Y, Levenson RM et al (2004) In vivo cancer targeting and imaging with semiconductor quantum dots. Nat Biotechnol 22:969-976

24. Kim S, Lim YT, Soltesz EG et al (2004) Near-infrared fluorescent type II quantum dots for sentinel lymph node mapping. Nat Biotechnol 22:93-97

25. Chen C, Peng J, Xia HS et al (2009) Quantum dots-based immunofluorescence technology for the quantitative determination of HER2 expression in breast cancer. Biomaterials 30:2912-2918

26. Chen C, Xia HS, Gong YP et al (2010) The quantitative detection of total HER2 load by quantum dots and the identification of a new subtype of breast cancer with different 5 year prognosis. Biomaterials 31:8818-8825

27. Chen C, Sun SR, Gong YP et al (2011) Quantum dots-based molecular classification of breast cancer by quantitative spectroanalysis of hormone receptors and HER2. Biomaterials 32:7592-7599

28. Glazer ES, Curley SA (2010) Radiofrequency field-induced thermal cytotoxicity in cancer cells treated with fluorescent nanoparticles. Cancer 116:3285-3293 
29. Gonda K, Watanabe TM, Ohuchi N et al (2010) In vivo nanoimaging of membrane dynamics in metastatic tumor cells using quantum dots. J Biol Chem 285:2750-2757

30. Chen C, Peng J, Xia H et al (2010) Quantum-dot-based immunofluorescent imaging of HER2 and ER provides new insights into breast cancer heterogeneity. Nanotechnology 21:095101

31. Liu XL, Peng CW, Chen C et al (2011) Quantum dots-based double-color imaging of HER2 positive breast cancer invasion. Biochem Biophys Res Commun 409:577-582

32. Peng CW, Liu XL, Chen C et al (2011) Patterns of cancer invasion revealed by QDs-based quantitative multiplexed imaging of tumor microenvironment. Biomaterials 32:2907-2917

33. Fang M, Yuan JP, Peng CW et al (2013) Quantum dots-based in situ molecular imaging of dynamic changes of collagen IV during cancer invasion. Biomaterials 34:8708-8717

34. Peng CW, Tian Q, Yang GF et al (2012) Quantum-dots based simultaneous detection of multiple biomarkers of tumor stromal features to predict clinical outcomes in gastric cancer. Biomaterials 33:5742-5752

35. Edge SB, Byrd DR, Compton CC et al (2010) AJCC cancer staging manual, 7th edn. Springer, New York

36. Wang LW, Yang GF, Chen JM et al (2014) A clinical database of breast cancer patients reveals distinctive clinico-pathological characteristics: a study from central China. Asian Pac J Cancer Prev 15:1621-1626

37. Yang XQ, Chen C, Peng CW et al (2011) Quantum dot-based quantitative immunofluorescence detection and spectrum analysis of epidermal growth factor receptor in breast cancer tissue arrays. Int J Nanomed 6:2265-2273

38. Sun JZ, Chen C, Jiang G et al (2014) Quantum dot-based immunofluorescent imaging of Ki67 and identification of prognostic value in HER2-positive (non-luminal) breast cancer. Int $\mathbf{J}$ Nanomed 9:1339-1346

39. Morton DL, Wen DR, Wong JH et al (1992) Technical details of intraoperative lymphatic mapping for early stage melanoma. Arch Surg 127:392-399

40. Helle M, Cassette E, Bezdetnaya L et al (2012) Visualisation of sentinel lymph node with indium-based near infrared emitting Quantum Dots in a murine metastatic breast cancer model. PLoS ONE 7:e44433

41. Pons T, Pic E, Lequeux N et al (2010) Cadmium-free CuInS2/ $\mathrm{ZnS}$ quantum dots for sentinel lymph node imaging with reduced toxicity. ACS Nano 4:2531-2538

42. Pic E, Pons T, Bezdetnaya L et al (2010) Fluorescence imaging and whole-body biodistribution of near-infrared-emitting quantum dots after subcutaneous injection for regional lymph node mapping in mice. Mol Imaging Biol 12:394-405

43. Kosaka N, Ogawa M, Sato N et al (2009) In vivo real-time, multicolor, quantum dot lymphatic imaging. J Invest Dermatol 129:2818-2822

44. Robe A, Pic E, Lassalle HP et al (2008) Quantum dots in axillary lymph node mapping: biodistribution study in healthy mice. BMC Cancer 8:111

45. Ballou B, Ernst LA, Andreko S et al (2007) Sentinel lymph node imaging using quantum dots in mouse tumor models. Bioconjug Chem 18:389-396

46. Kobayashi H, Hama Y, Koyama Y et al (2007) Simultaneous multicolor imaging of five different lymphatic basins using quantum dots. Nano Lett 7:1711-1716

47. Hama Y, Koyama Y, Urano Y et al (2007) Simultaneous twocolor spectral fluorescence lymphangiography with near infrared quantum dots to map two lymphatic flows from the breast and the upper extremity. Breast Cancer Res Treat 103:23-28

48. Hama Y, Koyama Y, Urano Y et al (2007) Two-color lymphatic mapping using Ig-conjugated near infrared optical probes. J Invest Dermatol 127:2351-2356
49. Kobayashi H, Ogawa M, Kosaka N et al (2009) Multicolor imaging of lymphatic function with two nanomaterials: quantum dot-labeled cancer cells and dendrimer-based optical agents. Nanomedicine 4:411-419

50. Zhang H, Zeng X, Li Q et al (2009) Fluorescent tumour imaging of type I IGF receptor in vivo: comparison of antibody-conjugated quantum dots and small-molecule fluorophore. $\mathrm{Br} \mathrm{J}$ Cancer 101:71-79

51. Chen LD, Liu J, Yu XF et al (2008) The biocompatibility of quantum dot probes used for the targeted imaging of hepatocellular carcinoma metastasis. Biomaterials 29:4170-4176

52. Rizvi SB, Rouhi S, Taniguchi S et al (2014) Near-infrared quantum dots for HER2 localization and imaging of cancer cells. Int J Nanomedicine 9:1323-1337

53. Tada H, Higuchi H, Wanatabe TM et al (2007) In vivo real-time tracking of single quantum dots conjugated with monoclonal antiHER2 antibody in tumors of mice. Cancer Res 67:1138-1144

54. Balalaeva IV, Zdobnova TA, Krutova IV et al (2012) Passive and active targeting of quantum dots for whole-body fluorescence imaging of breast cancer xenografts. J Biophotonics 5:860-867

55. Papagiannaros A, Levchenko T, Hartner W et al (2009) Quantum dots encapsulated in phospholipid micelles for imaging and quantification of tumors in the near-infrared region. Nanomedicine 5:216-224

56. Takeda M, Tada $H$, Higuchi $H$ et al (2008) In vivo single molecular imaging and sentinel node navigation by nanotechnology for molecular targeting drug-delivery systems and tailormade medicine. Breast Cancer 15:145-152

57. Li Y, Li Z, Wang X et al (2012) In vivo cancer targeting and imaging-guided surgery with near infrared-emitting quantum dot bioconjugates. Theranostics 2:769-776

58. Ntziachristos V, Ripoll J, Weissleder R (2002) Would near-infrared fluorescence signals propagate through large human organs for clinical studies? Opt Lett 27:333-335

59. Ntziachristos V, Bremer C, Weissleder R (2003) Fluorescence imaging with near-infrared light: new technological advances that enable in vivo molecular imaging. Eur Radiol 13:195-208

60. Tan YF, Chandrasekharan P, Maity D et al (2011) Multimodal tumor imaging by iron oxides and quantum dots formulated in poly (lactic acid)-D-alpha-tocopheryl polyethylene glycol 1000 succinate nanoparticles. Biomaterials 32:2969-2978

61. Ma Q, Nakane Y, Mori Y et al (2012) Multilayered, core/shell nanoprobes based on magnetic ferric oxide particles and quantum dots for multimodality imaging of breast cancer tumors. Biomaterials 33:8486-8494

62. Ding K, Jing L, Liu C et al (2014) Magnetically engineered Cdfree quantum dots as dual-modality probes for fluorescence/magnetic resonance imaging of tumors. Biomaterials 35:1608-1617

63. SalmanOgli A, Rostami A (2012) Modeling and improvement of breast cancer site temperature profile by implantation of onionlike quantum-dot quantum-well heteronanocrystal in tumor site. IEEE Trans Nanotechnol 11:1183-1191

64. Park JH, von Maltzahn G, Ruoslahti E et al (2008) Micellar hybrid nanoparticles for simultaneous magnetofluorescent imaging and drug delivery. Angew Chem Int Ed Engl 47:7284-7288

65. Papagiannaros A, Upponi J, Hartner W et al (2010) Quantum dot loaded immunomicelles for tumor imaging. BMC Med Imaging 10:22

66. Gupta P, Adkins C, Lockman P et al (2013) Metastasis of breast tumor cells to brain is suppressed by phenethyl isothiocyanate in a novel metastasis model. PLoS ONE 8:e67278

67. de Boer M, van Deurzen CH, van Dijck JA et al (2009) Micrometastases or isolated tumor cells and the outcome of breast cancer. N Engl J Med 361:653-663

68. Ojima T, Kinami S, Nakamura K et al (2013) Advantages of the rapid double-staining method for intraoperative detection of micrometastasis in sentinel lymph nodes. Oncol Rep 30:1067-1072 
69. Kosaka N, Bernardo M, Mitsunaga M et al (2012) MR and optical imaging of early micrometastases in lymph nodes: triple labeling with nano-sized agents yielding distinct signals. Contrast Media Mol Imaging 7:247-253

70. Chan WH, Shiao NH (2008) Cytotoxic effect of CdSe quantum dots on mouse embryonic development. Acta Pharmacol Sin 29:259-266

71. Chu M, Wu Q, Yang H et al (2010) Transfer of quantum dots from pregnant mice to pups across the placental barrier. Small 6:670-678
72. Zhang Y, Hong G, Chen G et al (2012) Ag2S quantum dot: a bright and biocompatible fluorescent nanoprobe in the second near-infrared window. ACS Nano 6:3695-3702

73. Yang Z, Xu M, Liu Y et al (2014) Nitrogen-doped, carbon-rich, highly photoluminescent carbon dots from ammonium citrate. Nanoscale 6:1890-1895 